\title{
HIGH SENSITIVITY IMMUNOCHROMATOGRAPHIC TEST FOR DETECTION OF ILLICIT DRUGS IN ORAL FLUID: A FIGHT AGAINST DRUGS OF ABUSE
}

\author{
İsmail Hamit HANCI ${ }^{1}$, Büşra GÖRGÜN², Kübra KAYA ${ }^{2}$, Leyla Didem \\ $\mathrm{KOZACI}^{2,3}$ *
}

${ }^{1}$ Department of Forensic Sciences, Faculty of Medicine, Ankara University, Ankara, Turkey.
${ }^{2}$ Department of Translational Medicine, Institute of Health Sciences, Ankara Yildirim Beyazit University, Ankara, Turkey.
${ }^{3}$ Department of Medical Biochemistry, Faculty of Medicine, Ankara Yildirim Beyazit University, Ankara, Turkey.

Address for Correspondence: Prof. Dr. Leyla Didem KOZACI, E-mail: Idkozaci@yahoo.com

Received: 17.07.2020; Accepted: 22.12.2020; Available Online Date: 04.03.2021

(C) Copyright 2021 by Dokuz Eylül University, Institute of Health Sciences - Available online at https://dergipark.org.tr/en/pub/jbachs

Cite this article as: Hanci IH, Gorgun B, Kaya K, Kozacı LD. Endothelial Protein C Receptor Expression is Regulated by Sp1 Transcription Factor in Murine Microglia. J Basic Clin Health Sci 2021; 1: 49- 53.

\begin{abstract}
Purpose: Illicit drug use poses a great threat to the integrity of our society and can lead to major problems such as traffic and workplace accidents, homicides, theft, terrorism mongering and suicide to name a few. Among the variety of commonly used drugs-of-abuse, cannabis, cocaine, opiates, amphetamine and its derivatives have been the most reported. Oral fluid drug testing has been gaining interest as an accurate screening method for the detection of illicit drugs in suspects under influence. In this study, the accuracy of NARCOTEC oral fluid drug-detection system was evaluated in comparison with the cut-off values.

Methods: A handheld device (NARCOTEC version 01.02, Morpho Biotec Limited, London, UK), NARCOTEC test, was used to interpret a total of seven illegal drugs (benzodiazepines, benzodiazepines, opiates, methamphetamine / MDMA, amphetamine, $\triangle 9-\mathrm{THC}$ and cocaine) test results. For the positive control tests, the cassettes were opened and $80 \mu \mathrm{L}$ of each positive control solution was pipetted onto the appropriate sample pad lane and allowed 3-5 mins to migrate to the end of the nitrocellulose membrane before the cassettes were read by the handheld device. For the negative control tests, the negative control solutions were pipetted at $80 \mu \mathrm{L}$ to each of the three lanes without any distinction and allowed $3-5$ mins to flow before the cassettes were read by the handheld device (Figure 2). 40 positive and 40 negative control tests were performed and read by the handheld device. Finally, the accuracy of the analysis for each analyte was determined.

Results and conclusion: Positive and negative control samples were each assayed in 40 different replicates. Among the 80 tests, only BZD yielded one false-negative result within the positive control samples. In this study, NARCOTEC drug detection system was able to detect seven illicit drugs (benzodiazepines, opiates, methamphetamine/MDMA, amphetamine, $\triangle 9-\mathrm{THC}$ and cocaine) with a $99 \%$ accuracy and within as little as $5 \mathrm{~min}$. This indicates that NARCOTEC can be safely used for point-of-care drug tests including roadside tests, forensics screening, workplaces, immigration offices, air controllers, pilots and travel ports.
\end{abstract}

Key words: Illicit drugs, driving under influence, point-of-care (POC), lateral flow immunoassay. 


\section{INTRODUCTION}

Cannabis, cocaine, opiates, along with amphetamine and its derivatives are common drugs of abuse that have increasingly been detected in the blood/urine samples of drivers [1]. Consequently, it is a necessity to develop efficient and reliable on-site tests to identify users under the influence of these illicit drugs. Oral fluid testing has emerged as a promising pointof-care (POC) method to detect people under the influence of illicit drugs, with common usage in roadside detection by traffic police [2-4]. Since oral fluid can be easily and directly collected in a noninvasive manner, the risk of adulteration is precluded, and the results can be obtained within minutes. The use of special sampling devices also helps to minimize sample contamination and the risk of infection $[3,5]$.

Blood and oral fluid concentrations of drugs-of-abuse are correlated and started to increase shortly after drug-use. While oral fluid is indicative of recent drug use, urine offers a wider window for drug detection, which is not in direct correlation with blood-drug levels $[4,6]$. Blood sampling and consecutive toxicological analysis is mandatory in all cases when the suspect shows signs of drug-use and/or a drugs-of-abuse screening test is positive [3]. In Turkey, on-site tests to identify drugs of abuse in saliva have become more common in recent years [4].

NARCOTEC is a POC drug screening test that offers results within 5-7 min using an observed sample collection protocol. Advantages of using NARCOTEC include direct testing, minimalized adulteration risk, easy sample collection in the presence of an officer and rapid re-test if necessary. It is based on an immunoassay technique intended for use in the detection of cocaine (COC), amphetamine (AMP), opiate (OPI), methamphetamine (MET/MDMA), cannabinoid, (THC), and benzodiazepines (BZD). In this study, the accuracy of NARCOTEC to detect the presence or absence of illicit drugs in oral fluid was established based on the cut-off values of the device itself.

\section{METHODS}

\section{NARCOTEC test}

The NARCOTEC test consists of a collector swab to collect oral fluid, a drug test cassette (DRU-ID/01, Morpho Biotec Limited, London, UK), which encapsulates three test strips that can detect a total of seven illicit drugs (benzodiazepines, opiates, methamphetamine/MDMA, amphetamine, $\triangle 9-\mathrm{THC}$, and cocaine) and a handheld device (NARCOTEC version 01.02, Morpho Biotec Limited, London, UK) to interpret the test results (Figure 1). The cut-off limits for the test are given in (Table 1).

\section{Lateral flow immunoassay strip}

The lateral flow immunoassay (LFIA) strips, which are based on the competitive assay method, consist of a plastic backing on which a sample pad is mounted on top of a conjugate pad, the latter overlapping a nitrocellulose membrane with its end located under an absorbent pad [7]. The sample pad receives the oral fluid sample and delivers it to the conjugate pad which contains dried, gold-conjugated antibodies (COC/MET-MDMA or AMP/THC or OPI/BZD) that become re-solubilized upon contact with the oral fluid sample and travel along the nitrocellulose membrane with the help of capillary flow. Each nitrocellulose membrane (three different membranes in total) consists of two separate test lines coated with COCBSA/MET-BSA or AMP-BSA/THC-BSA or OPI$B S A / B Z D-B S A$ and one control line coated with goat anti-mouse IgG-BSA, all of which are immobilized with BSA to prevent them from migrating with the oral fluid sample. When a negative oral fluid sample is applied, the antibodies from the conjugate pad migrate along the nitrocellulose membrane and bind onto the test and control lines to produce red lines indicating a negative result. However, when an oral fluid sample spiked with a drug is applied, the drug binds to its specific antibody on the conjugate pad, preventing the gold-conjugated antibody from binding to the antigen-BSA on the test line, thus producing no line for that particular drug and consequently indicating a positive result. The appearance of the control line is a necessity to validate the functionality of the test, regardless whether the oral fluid sample contains illicit drug(s) or not. The absorbent pad serves to collect the oral fluid samples reaching the end of the nitrocellulose membrane and aids in maintaining continuous capillary flow for the duration of the test.

For the positive control tests, the cassettes were opened and $80 \mu \mathrm{L}$ of each positive control solution was pipetted onto the appropriate sample pad lane and allowed 3-5 mins to migrate to the end of the nitrocellulose membrane before the cassettes were 
read by the handheld device. For the negative control tests, the negative control solution was pipetted at 80 $\mu \mathrm{L}$ to each of the three lanes without any distinction and allowed 3-5 mins to flow before the cassettes

Table 1. NARCOTEC handheld device cut-off values

\begin{tabular}{cc}
\hline Substance & Cut-off $(\mathbf{n g} / \mathbf{m L})$ \\
\hline MET/MDMA & 50 \\
\hline AMP & 50 \\
\hline THC & 25 \\
\hline BZD & 20 \\
\hline COC & 30 \\
\hline OPI & 40 \\
\hline
\end{tabular}

Table 2. Negative and positive control standard solution drug concentrations

\begin{tabular}{ccc}
\hline \multirow{2}{*}{ Drug } & \multicolumn{2}{c}{ Standard Concentration $\mathbf{( n g} / \mathbf{m L})$} \\
\cline { 2 - 3 } & Negative Control & Positive Control \\
\hline MET/MDMA & 15 & 65 \\
\hline COC & 10 & 40 \\
\hline AMP & 15 & 65 \\
\hline THC & 10 & 35 \\
\hline BZD & 8 & 30 \\
\hline OPI & 15 & 50 \\
\hline
\end{tabular}

Table 3. Accuracy of NARCOTEC drug test system.

\begin{tabular}{cc}
\hline Test & Accuracy \\
\hline BZD $^{*}$ & $99 \%$ \\
\hline COC $^{*}$ & $100 \%$ \\
\hline MET $^{*}$ & $100 \%$ \\
\hline OPI $^{*}$ & $100 \%$ \\
\hline THC $^{*}$ & $100 \%$ \\
\hline AMP $^{*}$ & $100 \%$ \\
\hline Total $^{\#}$ & $100 \%$ \\
\hline Total $^{\$}$ & $100 \%$ \\
\hline Total $^{*}$ & $99 \%$ \\
\hline
\end{tabular}

\# Positive control

$\$$ Negative control

* Positive control +Negative control

\section{Control solutions}

The negative (Level 2; A3902) and positive (Level 5; A3902) control solutions were purchased from manufacturing company (AMS UK Ltd) in ready-touse form. Solutions consisted of oral fluid samples with an average $\mathrm{pH}$ of 7.4 and centrifuged at 10000 rpm for 15 mins at room temperature. The negative control solution contained (S)-(+)-Methamphetamine hydrochloride (MET, $15 \mathrm{ng} / \mathrm{mL}$, CAS no: 51-57-0, Chiron AS, Norway) and benzoylecgonine (COC, 10 ng/mL, CAS no: 519-09-5, Chiron AS, Norway), DLAmphetamine hydrochloride (AMP, $15 \mathrm{ng} / \mathrm{mL}$, CAS no: 2706-50-5, Chiron AS, Norway) and (-)-trans- $\Delta 9$ THC (THC, $10 \mathrm{ng} / \mathrm{mL}$, CAS no: 1972-08-3, Chiron
AS, Norway ), and oxazepam (BZD, $8 \mathrm{ng} / \mathrm{mL}$, CAS no: 604-75-1, Chiron AS, Norway) and morphine monohydrate (OPI, $15 \mathrm{ng} / \mathrm{mL}$, CAS no: 6009-81-0, Chiron AS, Norway). The positive control solution contained (S)-(+)-Methamphetamine hydrochloride (MET, $65 \mathrm{ng} / \mathrm{mL}$, CAS no: 51-57-0, Chiron AS, Norway) and benzoylecgonine (COC, $40 \mathrm{ng} / \mathrm{mL}$, CAS no: 519-09-5, Chiron AS, Norway), DL-Amphetamine hydrochloride (AMP, $65 \mathrm{ng} / \mathrm{mL}$, CAS no: 2706-50-5, Chiron AS, Norway) and (-)-trans- $\triangle 9-T H C$ (THC, 35 ng/mL, CAS no: 1972-08-3, Chiron AS, Norway ) and oxazepam (BZD, $30 \mathrm{ng} / \mathrm{mL}$, CAS no: 604-75-1, Chiron AS, Norway) and morphine monohydrate (OPI, $50 \mathrm{ng} / \mathrm{mL}$, CAS no: 6009-81-0, Chiron AS, Norway). The positive and negative control solutions were stored at $-20 \circ \mathrm{C}$ until used (Table 2).

\section{Accuracy calculation}

The accuracy of the analysis for each analyte was determined according to the formula below [7]:

$\%$ Accuracy $=\frac{\text { True Positive }+ \text { True Negative }}{\text { True Positive }+ \text { True Negative }+ \text { False Positive }+ \text { False Negative }} \times 100 \%$

\section{RESULTS AND DISCUSSION}

NARCOTEC on-site drug testing handheld device is conveniently used with a prepacked kit to collect the sample. Each kit contains 1 cassette and a saliva collection swab (Figure 3). The collector swab is placed under the tongue of the suspect and allowed on average $2 \mathrm{~min}$ to become saturated with oral fluid, which is signalled by a red colour change at the base of the plastic handle.

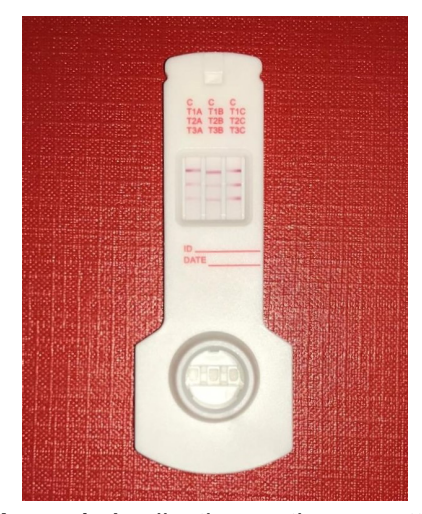

Figure 1. Application on the cassette.

This precludes the risk of sample tampering witnessed in urine drug tests where the suspect might dilute their urine with water to alter the test results and 


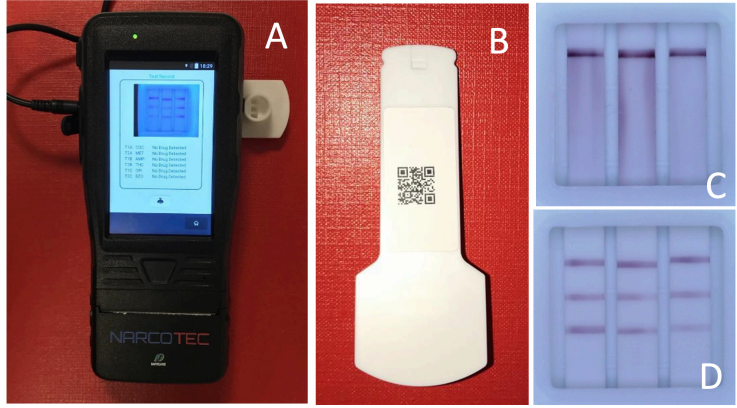

Figure 2. NARCOTEC Device (A), QR labelled cassette (B) and screens with positive $(C)$ and negative $(D)$ test results.

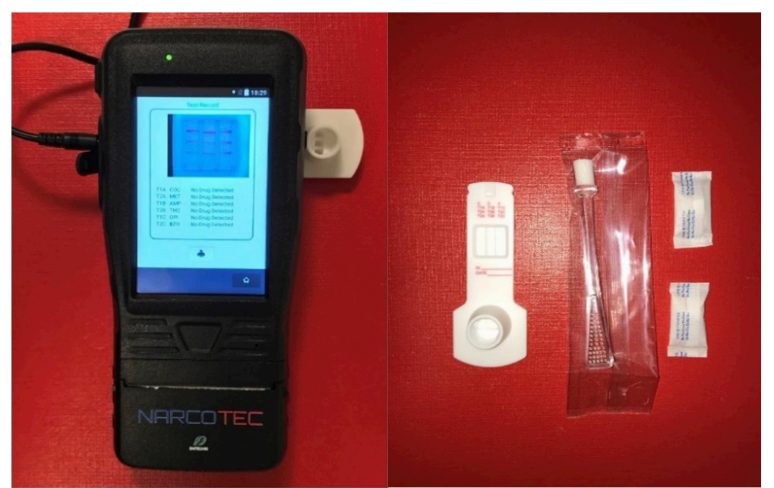

Figure 3. NARCOTEC handheld device and cassette kit.

is less invasive than blood drug tests, which can increase the stress level of subjects, and which are generally more challenging to perform especially on long-term drug abusers whose veins might be damaged. Then, the collector swab is pressed into the receiving area of the cassette to allow the oral fluid to be absorbed by the sample pad. The completion of the test takes $\sim 5 \mathrm{~min}$, after which the QR code found on the back of the cassette is read by the NARCOTEC handheld device, which also consists of a user-friendly touch screen from which the suspect's information can be logged in (Figure 2). The cassette is then inserted into the reading area for data interpretation, which takes $10-15 \mathrm{~s}$ in total. The handheld device utilizes a built-in camera that detects the presence or absence of the test lines and accordingly informs the user whether a laboratory test is required in the case of a positive result or no further tests are required in the case of a negative result. This eliminates the possibility of subjective assessment of results and further offers on-site test result printouts for archival and legal purposes. The test requires minimal training due to the straightforward nature of the technology and can be performed at any given site. This eliminates the need for highly trained lab technicians along with expensive lab equipment and techniques such as LC-MS-MS and ELISA, which will only be needed for the confirmation and quantification of positive on-site test results if detected [8]. Therefore, instead of routinely performing complicated analytical methods for the detection of drugs that might yield numerous negative test results, on-site drug tests can be performed as a preliminary screening procedure, before resorting to more complex methods, thus reducing manpower, time and expenses.

Positive and negative control samples were each assayed in 40 different replicates (Figure 1). Among the 80 tests, only BZD yielded one false-negative result within the positive control samples (Table 3 ). In the evaluation of the results, after determining true positive (TP), true negative (TN), false positive (TP) and false negative (TN) values, the accuracy rate of the NARCOTEC Device was calculated as $99 \%$. This was due to the false-negative result obtained by the BZD reading, which is still in the range of acceptable accuracy limits. One important issue in on-site drug testing is the accuracy of negative results and the low ratio of false-positive results. In this study, we observed $100 \%$ accuracy in concentrations below the cut-off values in all tests, i.e., negative control samples.

\section{CONCLUSION}

Oral fluid drug tests are reliable, rapid, non-invasive and easy-to-use preliminary screening tests that are capable of detecting suspects under the influence of illicit drugs. In this study, NARCOTEC drug detection system was able to detect seven illicit drugs (benzodiazepines, opiates, methamphetamine/MDMA, amphetamine, $\triangle 9$-THC and cocaine) with a $99 \%$ accuracy and within as little as $5 \mathrm{~min}$. This indicates that NARCOTEC can be safely used for point-of-care drug tests including roadside tests, forensics screening, workplaces, immigration offices, air controllers, pilots and travel ports.

Conflict of interest: The authors declare there is no conflict of interest.

Acknowledgments: The test cassettes were designed by Morpho Biotec Limited (London, UK) in collaboration with Marmara Teknokent (Istanbul, Turkey).

Peer-review: Externally peer-reviewed. 


\section{REFERENCES}

1. Ji Kwon N, Han E. A review of drug abuse in recently reported cases of driving under the influence of drugs (DUID) in Asia, USA, and Europe. Forensic Sci Int. 2019; 302: 109854.

2. Aps JK, Martens LC. Review: The physiology of saliva and transfer of drugs into saliva. Forensic Sci Int. 2005; 150(2-3): 119-31.

3. Blencowe T, Pehrsson A, Lillsunde P, Vimpari K, Houwing S, Smink B, Mathijssen R, Van der Linden T, Legrand SA, Pil K. Verstraete A. An analytical evaluation of eight on-site oral fluid drug screening devices using laboratory confirmation results from oral fluid. Forensic Sci Int. 2011; 208(1-3): 173-79.

4. Hancı İH, Kozaci LD. Determination of Drugs in Saliva in Combating Addictive Drugs: An Easy to Apply Immunochromographic Method. 2019; 3(4): 128-43.

5. Vindenes V, Lund HM, Andresen W, Gjerde H, Ikdahl SE, Christophersen AS, Øiestad EL. Detection of drugs of abuse in simultaneously collected oral fluid, urine and blood from Norwegian drug drivers. Forensic Sci Int. 2012; 219(1-3): 165-71.

6. Toennes SW, Kauert GF, Steinmeyer S, Moeller $M R$. Driving under the influence of drugs evaluation of analytical data of drugs in oral fluid, serum and urine, and correlation with impairment symptoms. Forensic Sci Int. 2005; 152(2-3): 14955.

7. Tang MHY, Ching CK, Poon S, Chan SSS, Ng WY, Lam M, Wong CK, Pao R, Lau A, Mak TWL. Evaluation of three rapid oral fluid test devices on the screening of multiple drugs of abuse including ketamine. Forensic Sci Int. 2018; 286: 113-20.

8. Šuláková A, Fojtíková L, Holubová B, Bártová K, Lapčík $O$, Kuchař $M$. Two immunoassays for the detection of $2 \mathrm{C}-\mathrm{B}$ and related hallucinogenic phenethylamines. J Pharmacol Toxicol Methods. 2019; 95: 36-46. 